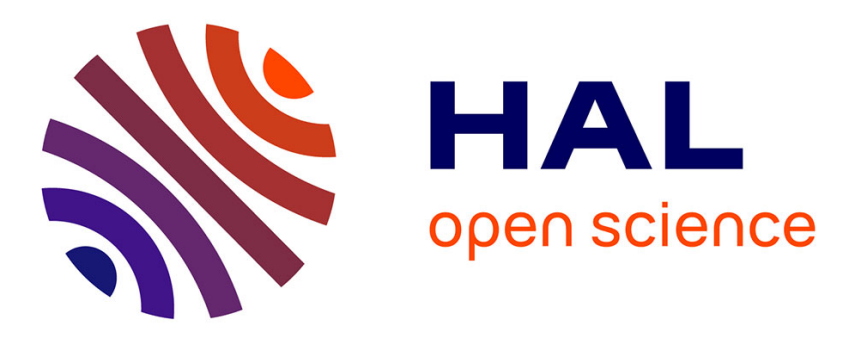

\title{
Modern Rutherford experiment: Tunneling of the most neutron-rich nucleus
}

\author{
A. Lemasson, A. Shrivastava, A. Navin, M. Rejmund, N. Keeley, V. \\ Zelevinsky, S. Bhattacharyya, Avhishek Chatterjee, G. de France, B. Jacquot, \\ et al.
}

\section{To cite this version:}

A. Lemasson, A. Shrivastava, A. Navin, M. Rejmund, N. Keeley, et al.. Modern Rutherford experiment: Tunneling of the most neutron-rich nucleus. Physical Review Letters, 2009, 103, 232701 (4 p.). 10.1103/PhysRevLett.103.232701 . in2p3-00433528

\section{HAL Id: in2p3-00433528 \\ https://hal.in2p3.fr/in2p3-00433528}

Submitted on 19 Nov 2009

HAL is a multi-disciplinary open access archive for the deposit and dissemination of scientific research documents, whether they are published or not. The documents may come from teaching and research institutions in France or abroad, or from public or private research centers.
L'archive ouverte pluridisciplinaire HAL, est destinée au dépôt et à la diffusion de documents scientifiques de niveau recherche, publiés ou non, émanant des établissements d'enseignement et de recherche français ou étrangers, des laboratoires publics ou privés. 


\title{
Modern Rutherford experiment: Tunneling of the most neutron-rich nucleus
}

\author{
A. Lemasson, ${ }^{1}$ A. Shrivastava,,${ }^{1,2}$ A. Navin, ${ }^{1,}{ }^{*}$ M. Rejmund, ${ }^{1}$ N. Keeley,${ }^{3}$ V. Zelevinsky, ${ }^{4}$ S. Bhattacharyya,${ }^{5}$ \\ A. Chatterjee, ${ }^{2}$ G. de France, ${ }^{1}$ B. Jacquot, ${ }^{1}$ V. Nanal, ${ }^{6}$ R. G. Pillay, ${ }^{6}$ R. Raabe, ${ }^{1}$ and C. Schmitt ${ }^{1}$ \\ ${ }^{1}$ GANIL, CEA/DSM - CNRS/IN2P3, Bd Henri Becquerel, BP 55027, F-14076 Caen Cedex 5, France \\ ${ }^{2}$ Nuclear Physics Division, Bhabha Atomic Research Centre, Mumbai 400085, India \\ ${ }^{3}$ Department of Nuclear Reactions, The Andrzej Sottan Institute for Nuclear Studies, ul. Hoża 69, PL-00-681 Warsaw, Poland \\ ${ }^{4}$ NSCL and Department of Physics and Astronomy, \\ Michigan State University, East Lansing, Michigan 48824-1321, USA \\ ${ }^{5}$ Variable Energy Cyclotron Centre, 1/AF Bidhan Nagar, Kolkata 700064, India \\ ${ }^{6}$ Department of Nuclear and Atomic Physics, Tata Institute of Fundamental Research, Mumbai 400005, India
}

\begin{abstract}
A modern variation of the Rutherford experiment to probe the tunneling of exotic nuclear matter from the measurement of the residues formed in the bombardment of ${ }^{197} \mathrm{Au}$ by extremely neutronrich ${ }^{8} \mathrm{He}$ nuclei is presented. Using a novel off-beam technique the most precise and accurate measurements of fusion and neutron transfer involving re-accelerated unstable beams are reported. The results show unusual behavior of the tunneling of ${ }^{8} \mathrm{He}$ compared to that for lighter helium isotopes, highlighting the role of the intrinsic structure of composite many-body quantum systems and pairing correlations.
\end{abstract}

PACS numbers: 25.70.Jj, 25.60.-t, 24.50.+g

The alpha particle, the most stable helium isotope, played a pioneering role in the discovery of the atomic nucleus and the first application of the theory of quantum tunneling. In addition to nuclear physics $[1,2]$, studies of the tunneling of composite objects [3-5] are of fundamental interest in molecular processes, transport in nanodevices and the physics of stellar evolution $[6,7]$. The fusion of complex nuclei, governed by a delicate balance between the attractive nuclear and repulsive Coulomb interactions, cannot be understood as simple barrier penetration by a structureless object with a potential depending only on the distance between the centers of the interacting systems. The tunneling probability is extremely sensitive to the plasticity of the intrinsic structure that can evolve during the process and to the interplay of many open and virtual channels [1, 8], whose amplitudes may be tuned by varying the beam energy and different projectile-target combinations. Short-lived Radioactive Ion Beams (RIB) with weak binding, unusual neutron/proton asymmetry and extended spatial distributions (halos), provide access to uncharted territories addressing fundamental questions of nuclear existence, multidimensional tunneling, pairing and cluster correlations $[9,10]$.

Measurements of fusion and transfer cross sections involving short-lived RIB around the Coulomb barrier are extremely restricted due to the required substantial increase in sensitivity [11] compared to stable beams. The helium isotopic chain, where the nucleon emission threshold varies from $20.5 \mathrm{MeV}$ to $0.9 \mathrm{MeV}$, provides unique opportunities for studying the effect of the intrinsic structure on the tunneling process. Both ${ }^{6} \mathrm{He}$ and ${ }^{8} \mathrm{He}$ have "Borromean" structures (removal of the alpha core or one of the neutrons makes the remaining system unbound).
Contrary to the general trend, the charge radius of ${ }^{8} \mathrm{He}$ is smaller than that of ${ }^{6} \mathrm{He}$ due to the more isotropic distribution of the four valence neutrons [12]. Strong di-neutron correlations [13] and the possibility of a dineutron condensate state [14] in ${ }^{8} \mathrm{He}$ have been suggested. The increase of the neutron separation energy in the more neutron-rich ${ }^{8} \mathrm{He}$ compared to ${ }^{6} \mathrm{He}$ is different from the known behaviour for all other nuclei. Both the experimental and theoretical complexities associated with ${ }^{8} \mathrm{He}$ make the study of the tunneling of this nucleus with the largest neutron/proton ratio a challenging problem. Here we report the first such measurements with re-accelerated beams of ${ }^{8} \mathrm{He}$ around the Coulomb barrier.

The RIB of ${ }^{8} \mathrm{He}\left(t_{1 / 2}=119.1 \mathrm{~ms}\right)$ were produced by fragmentation of a $75 \mathrm{MeV} /$ nucleon ${ }^{13} \mathrm{C}$ beam on a thick graphite target, then fully purified and re-accelerated by the CIME cyclotron at the SPIRAL facility at GANIL. The ${ }^{8} \mathrm{He}$ beam, with an energy resolution better than $2 \times 10^{-3}$ and a beam spot size of $\sim 5 \mathrm{~mm}$ diameter and an average intensity of $4 \times 10^{5}$ particles/s, bombarded a stack of ${ }^{197} \mathrm{Au}$ targets. The schematic of the setup is shown in Fig. 1. The target stacks consisted of two/three $\mathrm{Au}$ targets $\left(\sim 6 \mathrm{mg} / \mathrm{cm}^{2}\right.$ thick $)$ separated by $\mathrm{Al}$ foils $\left(\sim 1 \mathrm{mg} / \mathrm{cm}^{2}\right.$ thick $)$ to collect recoiling residues and $\mathrm{Al}$ foils (from 2 to $10 \mathrm{mg} / \mathrm{cm}^{2}$ thick) to degrade the beam energy. The different target stacks were irradiated at energies of 2.34, 2.51 and $3.68 \mathrm{MeV} /$ nucleon for $150 \mathrm{~h}, 24 \mathrm{~h}$ and $32 \mathrm{~h}$, respectively. The intensity of the ${ }^{8} \mathrm{He}$ beam was measured using a microchannel plate and a plastic scintillator $(10 \times 10 \times 0.05 \mathrm{~cm})$ placed respectively $10 \mathrm{~cm}$ upstream and $5 \mathrm{~cm}$ downstream with respect to the target (Fig. 1a). Their time-stamped energy and time signals, correlated with the cyclotron frequency, were used to measure the ${ }^{8} \mathrm{He}$ beam intensity and its time varia- 


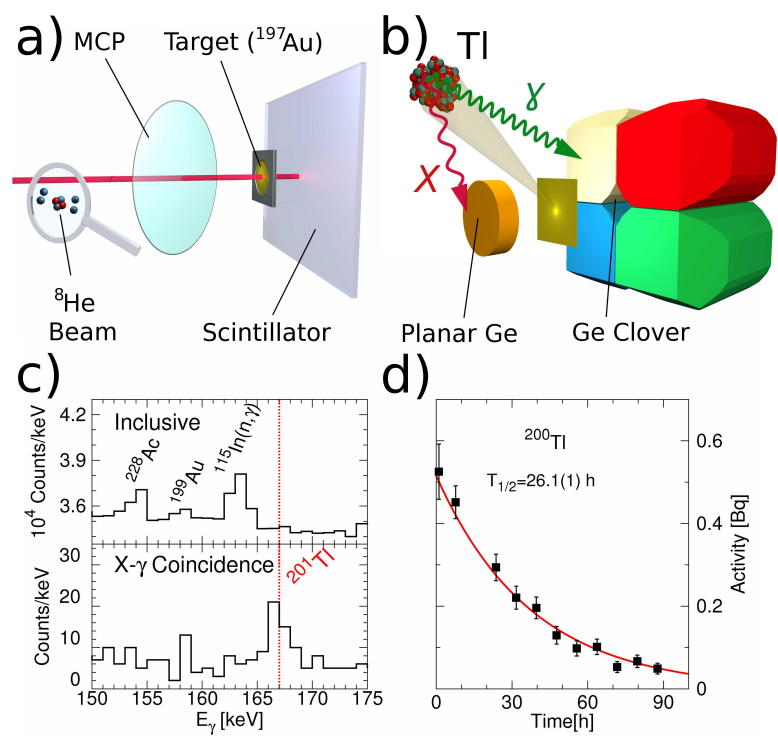

FIG. 1: (Color online) Experimental configuration: (a) Schematic view of the in-beam experimental setup: A microchannel plate detector (MCP) and a plastic scintillator placed before and after the target were used to measure the number of incident beam particles. (b) Schematic view of the off-beam experimental setup: Irradiated targets were placed between two HPGe detectors in face-to-face geometry in a $\mathrm{Pb}$ shielded enclosure to measure the radiation from decaying residues. (c) Gamma-ray spectra : Inclusive (top) and in coincidence (bottom) with characteristic $\mathrm{K}_{\alpha} \mathrm{X}$ rays of $\mathrm{Hg}$ (resulting from the decay of $\mathrm{Tl}$ ), at $E_{\mathrm{lab}}=16.7 \mathrm{MeV}$, illustrating the sensitivity of the new experimental technique. (d) ${ }^{200} \mathrm{Tl}$ activity (using the above coincidence) at $E_{\text {lab }}=22.9 \mathrm{MeV}$. The solid line shows the fitted activity using the known halflife.

tion during the irradiation. The incident beam energy on the successive target foils was determined based on the energy loss for the Al and Au foils using SRIM [15].

Various reactions can take place: fusion, neutron transfer, direct breakup and elastic scattering of the projectile. The ${ }^{199-202} \mathrm{Tl}$ nuclei, arising after the evaporation of neutrons from the compound nucleus ${ }^{205} \mathrm{Tl}$, were characterized by their radioactive decay. These off-beam measurements were made using two lead-shielded detector setups, the first optimized for X- $\gamma$ coincidences (Fig. 1b) and the second consisting of two clover detectors to maximize the $\gamma$-ray detection efficiency. Absolute photo-peak efficiencies of the above two setups were obtained using complete GEANT4 [16] simulations which reproduced within $4 \%$ the results of measurements made with five calibration sources (including a ${ }^{201} \mathrm{Tl}$ source) at various distances. Unambiguous identification of the energy and time of disintegration was obtained using a new sensitive and accurate off-beam coincidence technique specialized for the measurement of absolute evaporation residue cross sections involving low intensity RIB. This method, ap- plied earlier [17] to high intensity stable beams, involves the simultaneous measurement of $\mathrm{X}$ and $\gamma$ rays emitted in electron capture decays. Typical $\gamma$-ray spectra, obtained with and without an X-ray coincidence and the measured activity, Fig. 1c - 1d, highlight the selectivity and sensitivity of the method. The final gain in sensitivity, compared to an inclusive experiment, was found to be $\sim 3 \times 10^{4}$, yielding the first complete and most accurate (having the same quality as for stable beams) fusion cross sections ever measured with re-accelerated RIB. The smallest cross sections measured here (Fig. 2) using re-accelerated RIB, are comparable to the current measurement limits in nuclear physics, when scaled by the million times larger intensities available with stable beams.

Shown in Fig. 2a are the measured individual fusionevaporation residue cross sections, $\sigma_{\mathrm{ER}}$, as a function of the excitation energy together with predictions of Bohr's model of the statistical decay of an equilibrated compound nucleus [18]. The excellent agreement between the measured $\sigma_{\mathrm{ER}}$ and the calculations confirms that the residues arise from the complete fusion of ${ }^{8} \mathrm{He}$ and ${ }^{197} \mathrm{Au}$. The fusion cross section, $\sigma_{\mathrm{F}}$, obtained from the sum of the $\sigma_{\mathrm{ER}}$, is shown in Fig. 2b. The residues (isotopes of gold) formed after transfer of neutron(s) from the projectile were identified and their activity obtained using inclusive $\gamma$-ray measurements. The total transfer cross section, $\sigma_{n}$, obtained from the sum of the cross sections for ${ }^{198,198 m, 199} \mathrm{Au}$ residues is also shown.

Figure $2 \mathrm{~b}$ also shows the calculated fusion cross sections for tunneling through a single barrier, depending only on the inter-nuclear distance, calculated with a nuclear potential derived either from a global parameterization [19] (dotted) or from a microscopic double-folded potential using realistic densities (dashed). The difference between the two calculations demonstrates the effect of the extended neutron distribution of the ${ }^{8} \mathrm{He}$ nucleus. The dramatic decrease in the measured fusion cross section for energies at and above the barrier, along with a modest increase below the barrier (with respect to the dashed line) can be observed from the figure. The dominance of the transfer processes is seen from the magnitude of the measured $\sigma_{n}$ that are much larger than $\sigma_{\mathrm{F}}$ even at energies well above the barrier. Coupled-channels calculations $[8,20]$ using the code FRESCO [21] were performed to understand the influence of the direct, one-step transfer channels ${ }^{197} \mathrm{Au}\left({ }^{8} \mathrm{He},{ }^{6,7} \mathrm{He}\right){ }^{199,198} \mathrm{Au}$ on the fusion process. The $1 n$-transfer calculations were made ignoring the spin of the ${ }^{197} \mathrm{Au}$ core (to keep them tractable) with appropriate single-particle states and spectroscopic factors. For the 2n-transfer, based on semiclassical Qmatching conditions[19], transitions to states above the 1n-emission threshold but below that for 2 n-emission for ${ }^{199} \mathrm{Au}$ were included. A pure $2 \mathrm{n}+{ }^{197} \mathrm{Au}$ cluster structure with cluster-core relative angular momentum ranging from 0 to $5 \hbar$ was considered. The distorting potentials in 

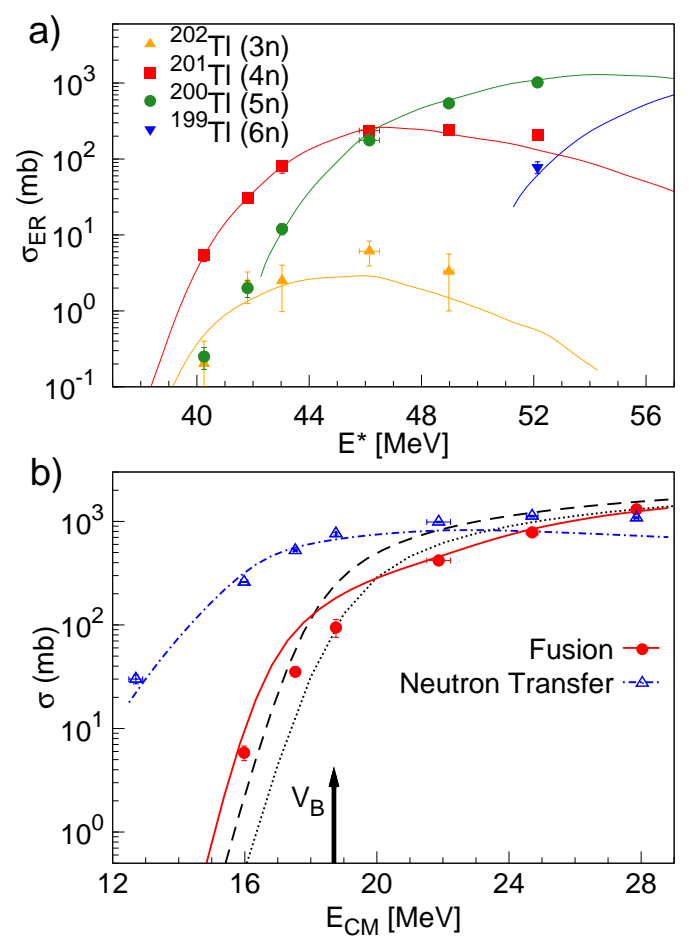

FIG. 2: (Color online) Cross sections for the ${ }^{8} \mathrm{He}+{ }^{197} \mathrm{Au}$ system: (a) The cross sections $\left(\sigma_{\mathrm{ER}}\right)$ for evaporation residues as a function of excitation energy of the compound nucleus. The lines correspond to predictions based on the statistical decay of an equilibrated compound nucleus [18]. (b) Fusion $\left(\sigma_{\mathrm{F}}\right)$ and neutron transfer $\left(\sigma_{n}\right)$ excitation functions. Two predictions for $\sigma_{\mathrm{F}}$ based on tunneling through a single barrier depending only on the radial separation are shown; dotted line - using a nuclear potential derived from a global parameterization [19]; dashed line - using a microscopic potential folding the nuclear densities of the target and the projectile. The coupled-channels calculations for fusion and transfer reactions are shown by continuous and dash-dotted lines, respectively. The nominal value of the Coulomb barrier $\left(V_{\mathrm{B}}\right)$ is indicated.

the entrance and exit channels consisted of double-folded real and short-ranged imaginary parts. The observed behavior of the fusion excitation function is reproduced by these coupled-channels calculations (continuous line) including coupling to the transfer channels (dash-dotted line). The good agreement of the calculations including only neutron transfer channels shows that the low particle threshold $(2.1 \mathrm{MeV})$ does not imply a crucial influence of breakup of the projectile on the tunneling process, as is generally assumed for weakly bound nuclei [8] (see Ref. [22] for a recent spectroscopic study of ${ }^{8} \mathrm{He}$ ). The role of pairing correlations which stabilize ${ }^{8} \mathrm{He}$ and result in an enhancement [2] of the neutron-pair transfer over single neutron transfer was also shown by the present calculations.

Interestingly, when juxtaposed to the calculations of the tunneling probability as pure barrier penetration (dotted line), the influence of the exotic intrinsic structure of ${ }^{8} \mathrm{He}$ on the tunneling probability is found to be relatively small compared to that observed in heavy-ion fusion $[1,23,24]$. With stable beams, the coupling of neutron transfer channels was shown to be related to an order of magnitude increase in $\sigma_{\mathrm{F}}$ below the Coulomb barrier $[23,24]$. In spite of large neutron transfer cross sections, the exotic nucleus ${ }^{8} \mathrm{He}$ unexpectedly displays a relatively weak fusion enhancement. This dramatic difference between ${ }^{8} \mathrm{He}$ and heavier systems could be attributed to the amplification of the relative changes in the barrier height by the exponential dependence of the tunneling probability.

The helium isotopic chain is a showcase for characteristic fingerprints of the features of tunneling of a composite object [3-5]. The ratio of masses, $\rho$, of interacting and non-interacting components (with respect to the Coulomb field) in a composite object, has been shown to affect the low-energy behaviour, increasing the tunneling probability [5] (as seen, for example, in deuterondeuteron reactions at $\mathrm{keV}$ energies [25]) and creating cusps and resonances [26]. The oscillations in the transmission coefficient have been shown to be damped with increasing $\rho$; such a result can also be inferred from Ref. [5]. In the neutron-rich helium isotopes the valence nucleons outside the alpha core can be treated as the "noninteracting" analog given the tight binding and point-like behavior of the alpha particle. The helium chain offers a unique progression of $\rho$ varying from $1\left({ }^{8} \mathrm{He}\right)$ and $2\left({ }^{6} \mathrm{He}\right)$ to the infinite limit for ${ }^{4} \mathrm{He}$. In such a picture the helium isotopes are expected to behave differently with respect to their barrier penetration.

Figure 3 shows the measured fusion cross sections for helium isotopes with ${ }^{197} \mathrm{Au}$. The good agreement between the calculated and measured fusion cross section for ${ }^{4} \mathrm{He}$ reiterates its point-like behavior. At energies below the barrier, $\sigma_{\mathrm{F}}$ for ${ }^{8} \mathrm{He}$ and ${ }^{6} \mathrm{He}[28]$ are unusually similar and as expected are larger than for ${ }^{4} \mathrm{He}$ [29]. A loosely bound system with a subsystem that does not feel the barrier can more easily restructure during the dynamical process of fusion, emphasizing the role of a flexible intrinsic wave function that can adiabatically readjust in a slow process and increase penetration [3]. Similar restructuring effects were predicted for the inverse processes of cluster knock-out [30]. The observed similarity of the low-energy results for ${ }^{8} \mathrm{He}$ and ${ }^{6} \mathrm{He}$ may indicate the role of higher order processes with neutron pair transfer preceding fusion. At energies well above the barrier, the fusion process is essentially "sudden" so the intrinsic configuration does not have time to change and $\sigma_{\mathrm{F}}$ is expected to be lower compared to an adiabatic approach. The most accurate and complete measurements of ${ }^{8} \mathrm{He}+{ }^{197} \mathrm{Au}$ fusion and transfer seem to indicate that the low-density neutron matter in the skin of ${ }^{8} \mathrm{He}$ is stabilized by pairing correlations. For a loosely bound but 


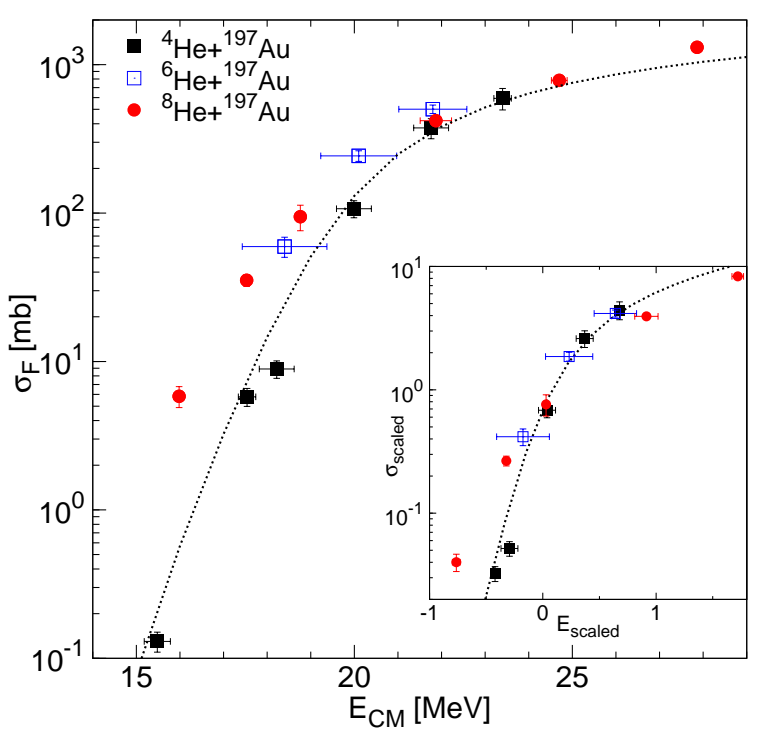

FIG. 3: (Color online) Measured fusion cross section as a function of center-of-mass energy $\left(E_{\mathrm{CM}}\right)$ for ${ }^{4,6,8} \mathrm{He}+{ }^{197} \mathrm{Au}$ systems. The data for ${ }^{4,6} \mathrm{He}+{ }^{197} \mathrm{Au}$ systems are from Refs. $[28,29]$. The dotted line shows the one-dimensional barrier penetration calculation for ${ }^{4} \mathrm{He}+{ }^{197} \mathrm{Au}$ obtained using the parameterization of Ref. [19] for the nuclear potential. The inset shows suitably scaled cross sections $\left(\sigma_{\text {scaled }}\right)$ and energies $\left(E_{\text {scaled }}\right)$ as suggested in Ref. [27], accounting for trivial differences in the height, radius and curvature of the barrier.

essentially isotropic system like ${ }^{8} \mathrm{He}$, it turns out to be easier to transfer part of the neutron excess in a peripheral reaction than to readjust the outer skin of the system and tunnel as a whole, a process which occurs only in a narrow region of small impact parameters. Understanding of the intriguing behavior of the helium isotopes will influence future applications towards the production of super-heavy elements [31] and the study of decoherence effects in open quantum systems [32]. Currently, such studies with RIB have been to a large extent limited to light nuclei near the drip line. Apart from technological challenges, the key to fulfilling the aspirations of synthesizing new elements and finding a new island of stability in the periodic table could lie in the use of higher- $Z$ halo nuclei with valence neutron(s) in $\ell=0$ orbitals so as to maximize the spatial extent of the wave function and hence lower the Coulomb barrier.

We thank V. Meot for providing the ${ }^{201} \mathrm{Tl}$ source, Yu. E. Penionzhkevich for providing ${ }^{6} \mathrm{He}+{ }^{197} \mathrm{Au}$ data in tabular form and Y. Blumenfeld for discussions. We would like to acknowledge the GANIL staff for obtaining the highest quality and intensity of re-accelerated ${ }^{8} \mathrm{He}$ beams and for all technical aspects and especially thank J. Ropert and G. Fremont for their technical contributions to the experimental setups. V.Z. acknowledges NSF grant
PHY-0758099. A.L. was partly supported by the Region Basse-Normandie (France).

* Corresponding author email : navin@ganil.fr

[1] A. B. Balantekin and N. Takigawa, Rev. Mod. Phys. 70, 77 (1998)

[2] G. Potel et al., Phys. Rep. (in press) (available http://arxiv.org/abs/0906.4298) (2009).

[3] C. A. Bertulani,V. V. Flambaum, and V. G. Zelevinsky. J. Phys. G 34, 2289 (2007).

[4] S. Bacca and H. Feldmeier, Phys Rev. C 73, 054608 (2006).

[5] M. R. A. Shegelski, J. Hnybida, and R. Vogt, Phys. Rev. A 78, 062703 (2008).

[6] K. Langanke, F.K. Thielemann and M. Wiescher, Nuclear Astrophysics and Nuclei Far from Stability, Lect. Notes Phys. 651 (Springer, Berlin, 2004) p. 383.

[7] M. Terasawa et al., Astrophys. J. 562, 470 (2001).

[8] N. Keeley, R. Raabe, N. Alamanos, and J. L Sida, Prog. Part. Nucl. Phys. 59, 579 (2007).

[9] D. F. Geesaman, C. K. Gelbke, R. V. F. Janssens, and B. M. Sherrill, Ann. Rev. Nucl. Part. Sci. 56, 53 (2006).

[10] B. M. Sherrill, Science 320, 751 (2008).

[11] R. F. Casten, Nuclear Structure From a Simple Perspective (Oxford Univ. Press, New York, second edition, 2000) p. 426.

[12] P. Mueller et al., Phys. Rev. Lett. 99, 252501 (2007).

[13] K. Hagino, N. Takahashi, and H. Sagawa, Phys. Rev. C 77, 054317 (2008).

[14] Y. Kanada-Enyo, Phys. Rev. C 76, 044323 (2007).

[15] J.F. Ziegler, The Stopping and Range of Ions in Matter. http://www.srim.org/.

[16] S. Agostinelli et al., Nucl. Instr. Meth. A 506, 250 (2003).

[17] A. Lemasson et al., Nucl. Instr. Meth. A 598, 445 (2009).

[18] F. Pülhofer, Nucl. Phys. A 280, 267 (1977).

[19] R. A. Broglia and A. Winther, Heavy Ion Reactions, Frontiers in Physics Lecture Note Series Vol. 84 (Addison-Wesley, Redwood City, CA, 1991).

[20] N. Keeley, N. Alamanos, K. W. Kemper, and K. Rusek, Prog. Part. Nucl. Phys. 63, 396 (2009).

[21] I. J. Thomson, Comput. Phys. Rep. 7, 167 (1988).

[22] M. S. Golovkov et al., Phys. Lett. B 672, 22 (2009).

[23] R. A. Broglia, C. H. Dasso, S. Landowne, Phys. Rev. C 32, 1426 (1985).

[24] A. M. Stefanini et al., Phys. Rev. C 76, 014610 (2007).

[25] H. Yuki et al., JETP Lett. 68, 823 (1998).

[26] N. Ahsan and A. Volya, Nuclear Many-Body Physics Where Structure and Reactions Meet (World Scientific, 2008) p. 539.

[27] L. F. Canto et al., J. Phys. G 36, 015109 (2009).

[28] Yu. E. Penionzhkevich et al., Eur. Phys. J. A 31, 185 (2007).

[29] M. S. Basunia, H. A. Shugart, A. R. Smith, and E. B. Norman, Phys. Rev. C 75, 015802 (2007).

[30] A. Sakharuk, V. Zelevinsky, and V. G. Neudatchin, Phys. Rev. C 60, 014605 (1999).

[31] Y. Aritomo, Phys. Rev. C 75, 024602 (2007).

[32] N.D. Antunes, F.C. Lombardo, D. Monteoliva, and P.I. Villar, Phys. Rev. E 73, 066105 (2006). 